\title{
Bound and resonant impurity states in a narrow gaped armchair graphene nanoribbon.
}

\author{
B. S. Monozon \\ Physics Department, Marine Technical University, 3 Lotsmanskaya Str., \\ 190008 St.Petersburg, Russia, \\ P. Schmelcher \\ Zentrum für Optische Quantentechnologien, Universität Hamburg, \\ Luruper Chaussee 149, 22761 Hamburg, Germany
}

November 14, 2017

\begin{abstract}
An analytical study of discrete and resonant impurity quasi-Coulomb states in a narrow gaped armchair graphene nanoribbon (GNR) is performed. We employ the adiabatic approximation assuming that the motions parallel ("slow") and perpendicular ("fast") to the boundaries of the ribbon are separated adiabatically. The energy spectrum comprises a sequence of series of quasi-Rydberg levels relevant to the "slow" motion adjacent from the low energies to the size-quantized levels associated with the "fast" motion. Only the series attributed to the ground size-quantized sub-band is really discrete, while others corresponding to the excited subbands consist of quasi-discrete (Fano resonant) levels of non-zero energetic widths, caused by the coupling with the states of the continuous spectrum branching from the low lying sub-bands. In the two- and three-subband approximation the spectrum of the complex energies of the impurity electron is derived in an explicit form. Narrowing the GNR leads to an increase of the binding energy and the resonant width both induced by the finite width of the ribbon. Displacing the impurity centre from the midpoint of the GNR causes the binding energy to decrease while the resonant width of the first excited Rydberg series increases. As for the second excited series their widths become narrower with the shift of the impurity. A successful comparison of our analytical results with those obtained by other theoretical and experimental methods is presented. Estimates of the binding energies and the resonant widths taken for the parameters of typical GNRs show that not only the strictly discrete but also the some resonant states are quite stable and could be studied experimentally in doped GNRs.
\end{abstract}




\section{Introduction}

The electron properties of two-dimensional (2D) graphene, a single-layer carbon sheet, has attracted much attention by both theoreticians and experimentalists (see [1] and references therein). Along with this related structures, namely graphene nanoribbons, are also under intensive investigation [2]. One of the reason for this is that the long electron mean free path in graphene up to $1 \mu \mathrm{m}$ opens a field of carbon-based nanoelectronics, where GNRs are used as interconnects in nanodevices. The unique electron mobility in graphene structures is caused by the strong bonding between the carbon atoms in the honeycomb lattice of graphene. This in turn prevents the replacing of the carbon atoms by alien ones. Nevertheless, graphene is not immune to extrinsic disorder and its transport properties [3] are very sensitive to impurities and defects [4].

The theoretical problem of an impurity in 2D graphene was considered originally in [5, 6, 7, 8, 9. In the vicinity of the Dirac points in $\vec{k}$ space, which are peaks of the double cones of the Fermi surface, the low-energy electronic excitations in gapless graphene are described by the equation of the effective mass approximation, which is formally identical to the 2D Dirac equation for a massless neutrino, having the Fermi speed $v_{F}=10^{6} \mathrm{~m} / \mathrm{c}$. In the presence of an attractive impurity centre of charge $Z$ screened by a medium of the effective dielectric constant $\epsilon_{\text {eff }}$ the electron states are drastically different for the subcritical $J_{c}<J$ and supercritical $J_{c}>J$ regions of the strength of the Coulomb interaction, where $J=|j| \hbar,|j|=1,2, \ldots$ and $J_{c}=Z e^{2} / 4 \pi \epsilon_{0} \epsilon_{\text {eff }} v_{F}$ are the 2D momentum of the impurity electron and that, having the speed $v_{F}$, respectively. Clearly, the super- and subcritical regimes can be reached for the dimensionless Coulomb potential strength $q=Z e^{2} / 4 \pi \varepsilon_{0} \epsilon_{\text {eff }} \hbar v_{F}$ for $q>|j|$ and $q<|j|$, respectively. The difference of the subcritical and supercritical electron states is caused by their different behaviour in the vicinity of the impurity centre $\vec{r} \longrightarrow 0$. The subcritical regime admits regular solutions to the Dirac equations, while the wave functions corresponding to the supercritical case oscillate and do not have any definite limit. The physical reason for this is that at the subcritical strengths $g<|j|$ the centrifugal potential barrier prevents the electron "fall to the centre" [10, while the supercritical strengths $g>|j|$ provide the collapse. Clearly, the continuum approach based on the Dirac formalism becomes inapplicable. The lattice-scale physics dominates that in turn requires a regularization procedure, namely the cutoff of the Coulomb potential at short distances $r_{0} \simeq a$, where $a \simeq 1.42 \AA$ is the $\mathrm{C}-\mathrm{C}$ distance in graphene. The physics of the supercritical impurity electron in graphene [6] closely resembles that of the relativistic electron in an atom having the nuclear charge $Z>137$ [11, 12, 13, 14]. Since, as it follows from below, only the supercritical regime is relevant to the impurity state in GNR, we focus on this case.

Numerical and analytical approaches developed on the tight-binding model of the graphene lattice and of the Dirac equation, subject to the regularization procedure, respectively, undertaken originally by Pereira [7] et.al. have revealed

the infinite number of the quasi-bound states, having the finite width, arising, as it was shown quasi-classically [9], from the collapsed states. If the require- 
ment of the regularity of the wave functions in the vicinity of the source of the electron attraction is to be replaced by the less rigorous condition of its square integrability an infinite number of the strictly discrete energy levels were found to occur. The Coulomb potential cutoff in the gapped [15] and no cutoff in the gapless graphene [16] induce the energy series bounded and unbounded from below, respectively.

In the GNR, which in principle can be treated as a quasi-1D structure, we can expect completely different results. The strictly discrete bound states regular at the impurity centre $(\vec{r}=0)$ are realized without the regularization procedure, in particular without the cutoff of the Coulomb potential, preventing the collapse. Consequential concerns lie in the well known fact that the reduction of the dimension of the structure increases the stability of the impurity electron. In units of the impurity Rydberg constant $R y$ the binding energy $E_{b}$ of the impurity electron in $3 \mathrm{D}$ bulk material is $E_{b}=1$, in the narrow $2 \mathrm{D}$ quantum well $E_{b}=4$ 17, and in the thin quantum wire of radius $R$ much less than the impurity Bohr radius $a_{0}, E_{b} \sim \ln ^{2}\left(R / a_{0}\right)$ [18. Besides, an extremely weak 3D atomic potential not providing bound electron states, transforms in the presence of a magnetic field into a quasi-1D system binding the electron [19]. It is relevant to note that these atomic states arise under as weak as one likes magnetic fields i.e. the as large as one likes magnetic lengths playing the same role as the width of the GNR. Note that the confinement attributed to the semiconductor thin films 20] replaces the 3D Coulomb potential $\left(\sim r^{-1}\right)$ by the effective 2D potential of the weaker singularity of the logarithmic character. It seems that in the quasi1D GNR the effect of the attenuation of the potential singularity preventing the fall to the centre exceeds that of the vanishing of the $2 \mathrm{D}$ centrifugal potential barrier promoting the collapse.

Clearly a study of the impurity electron state in graphene structures is important on account of two reasons. First, these structures provide a realization in solid state physics of remarkable effects of quantum electrodynamics caused by a large "fine structure constant" $e^{2} / \hbar v_{F} \simeq 2.5$ [8, 9, 21, 22]. Second, we expect a strong impact of impurities on the electronic systems not only for $2 \mathrm{D}$ graphene layers possessing an outstanding high electron mobility [6] but in particular for impurity GNRs whose properties are not widely addressed in the literature yet.

Brey and Fertig 23 have shown that the energy spectrum of the electron in an armchair GNR bounded in $x$-direction is the sequence of the subbands formed by the branches of the continuous energies of the longitudinal unbounded $y$-motion emanating from the size-quantized energy levels $\varepsilon_{N},(N$ is the discrete lable), reflecting the ribbons $x$-confinement. The equation for the components $u_{A, B}(y)$ of the Dirac spinor relevant to the A and B sublattices of the graphene for the electron positioned far away from the impurity centre has the form

$$
u_{A, B}^{\prime \prime}(y)+\frac{E^{2}-\varepsilon_{N}^{2}}{\hbar^{2} v_{F}^{2}} u_{A, B}(y)=0,
$$

showing that the armchair GNR manifests itself as gapped structure entailing 


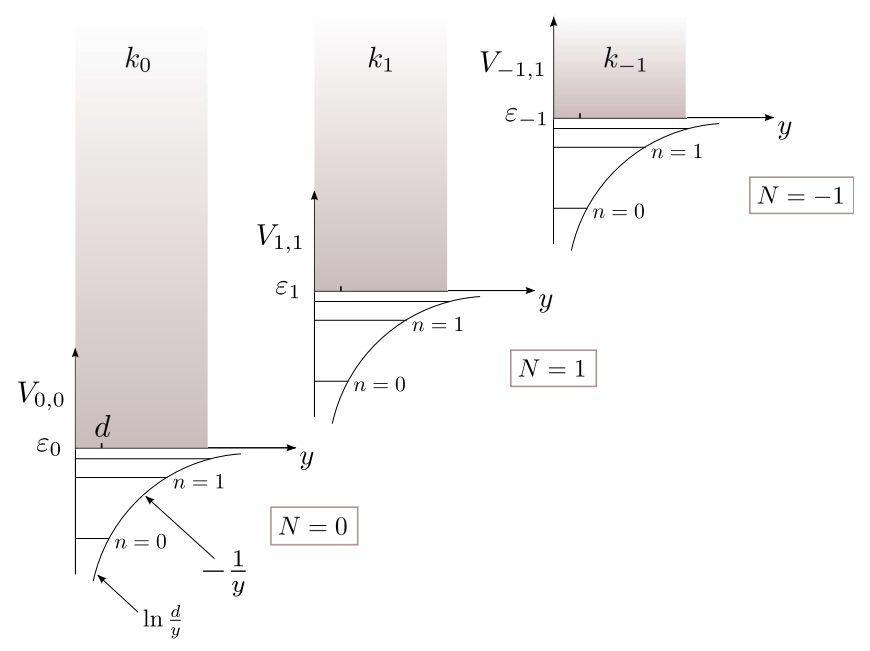

Figure 1: A schematic form of the potentials $V_{N N}(y)$ provided in eqs. (2), (11), (14) at $x_{0}=0$ and quasi-discrete $n$ (26) and continuous $k_{N}$ (29) spectra adjacent to the ground $N=0$ (discrete states) and first $N=1$ and second $N=-1$ size-quantized levels $\varepsilon_{N}$ (7) in the GNR of width $d$.

the bound and unbound impurity states for the energies $E^{2}<\varepsilon_{N}^{2}$ and $E^{2} \geq \varepsilon_{N}^{2}$, respectively.

Of special interest is the narrow GNR of width $d$ for which $d<<r_{0}$, where $r_{0}^{2}(d) \simeq \hbar^{2} v_{F}^{2}\left(E^{2}-\varepsilon_{N}^{2}\right)^{-1}$ is the radius of the bound electron state, being induced by the ribbon confinement $d$. Such a GNR provides the expected electron binding energy $E_{b} \sim \varepsilon_{N} f(q)$, where $f(q)$ is a some function vanishing at $q=0$, which is of interest and attractive because of two aspects. On the one hand the ribbon provides a considerable impurity binding energy which could be measured experimentally and on the other hand the impurity potential can be treated perturbatively and an analytical approach to the problem becomes feasible.

A comment concerning the from of the energy spectrum is in order. In the zeroth approximation of isolated size-quantized $N$-subbands i.e. in the singlesubband approximation the slow longitudinal motion parallel to the boundaries is governed by the $2 \mathrm{D}$ Coulomb potential averaged with respect to the "fast" transverse $N$-states. The energy spectrum consists of sequence of series of quasi-Coulomb discrete $\mathrm{Nn}$-levels and continuous sub-bands positioned below and above, respectively relatively to the size-quantized energy levels $\varepsilon_{N}$ (see Fig. 1). Only the series of the impurity energy levels $E_{0 n}<\varepsilon_{0}$ adjacent to the ground size-quantized energy level $\varepsilon_{0}$ is strictly discrete. The $N n$-series adjacent to the excited levels $N>0$ come into resonance with the states of the continuous spectra of lower subbands and in fact in the next multi-subband approximation turn into quasi-discrete resonant states (Fano resonances) 24]. 
The corresponding resonant widths $\Gamma_{N n}$ determine the auto-ionization rate and life-time $\tau_{N n}=\hbar / \Gamma_{N n}$ of the resonant impurity states being of relevance to an experimental study. Also to our knowledge an analytical approach to the problem of impurities in GNR providing the explicit dependencies of both discrete and especially quasi-discrete electron states on the width of the GNR $(d)$ and the position of the impurity centre within the GNR are not comprehensively available in the literature.

In order to fill the above mentioned gap we perform an analytical study of the strictly discrete and resonant impurity states in a narrow armchair GNR. The impurity centre is positioned anywhere within the ribbon bound by the impenetrable boundaries. The width of the GNR is assumed to be much less than the radius of the impurity state. The complete $2 \mathrm{D}$ envelope wave function satisfying the massless Dirac equation is expanded with respect to the basis formed by the $1 \mathrm{D}$ size-quantized subband wave functions describing the fast transverse motion bound by the boundaries of the GNR. The generated set of equations for the $1 \mathrm{D}$ quasi-Coulomb wave functions relevant to the longitudinal slow motion is solved in the single-, two- and three-subband approximations, in which the ground, first and second excited subbands are involved. The mathematical method is based on the matching of the Coulomb wave functions with those obtained by an iteration procedure at any point within the intermediate region bound by the ribbon width and the radius of the created Coulomb state. Both the real and imaginary parts of the complex energy levels are calculated in a single procedure. The dependencies of the binding energy and resonant energy shift and width on the width of the GNR and the position of the impurity centre are obtained in an explicit form. Numerical estimates show that for a narrow GNR the binding energy and the resonant width are quite reasonable, to render the impurity electron states in GNR experimentally observable. Our analytical results are in line with those calculated numerically and revealed in an experiment. We remark that our aim is to elucidate the physics of the impurity states in GNR by deriving closed form analytical expressions for their properties. We do not intend to compete with the results of computational studies.

The paper is organized as follows. In Section 2 the analytical approach based on the multi-subband approximation is described. The real quasi-Coulomb functions of the discrete and continuous spectrum and the real energy levels determining the binding energies are calculated in the single-subband approximation in Section 3. The complex energies including the resonant shift and width associated to the first and second excited subbands are found in the double and three-subband approximation, respectively, in Section 4. In Section 5 we discuss the obtained results. Section 6 contains the conclusions.

According to the above an analytical description of the stable and metastable impurity electron states in the narrow armchair GNR is of significant interest. It elucidates the underlying basic physics of the carbon-based nanodevices, in which the highly mobile electrons remain unbound in the 2D graphene monolayers while in their interconnects, namely in quasi-1D armchair GNRs these electrons are trapped by impurity centres. The latter could modify the overall transport properties. 


\section{General approach}

We consider a ribbon of width $d$ located in the $x-y$ plane and bounded by the lines $x= \pm d / 2$. The impurity centre of charge $Z$ is displaced from the mid-point of the ribbon $x=0$ by the distance $-d / 2 \leq x_{0} \leq d / 2$. The equation describing the impurity electron at a position $\vec{\rho}=(x, y)$ possesses the form of a Dirac equation

$$
\hat{\mathrm{H}}(\hat{\vec{k}}, \vec{\rho}) \vec{\Psi}(\vec{\rho})=E \vec{\Psi}(\vec{\rho}) ; \quad \hat{\vec{k}}=-i \vec{\nabla}
$$

where the Hamiltonian $\hat{\mathrm{H}}$ is given by

$$
\hat{\mathrm{H}}=p\left[\hat{\mathrm{H}}_{0}\left(\hat{k}_{x}\right)+\hat{\mathrm{H}}_{1}\left(\hat{k}_{y}\right)\right]+V(\vec{\rho}) \hat{\mathrm{I}} ; p=\hbar v ; v=10^{6} \mathrm{~m} / \mathrm{c}
$$

with

$$
\hat{\mathrm{H}}_{0}\left(\hat{k}_{x}\right)=\left(\begin{array}{cc}
-\sigma_{x} \hat{k}_{x} & 0 \\
0 & \sigma_{x} \hat{k}_{x}
\end{array}\right) ; \quad \hat{\mathrm{H}}_{1}\left(\hat{k}_{y}\right)=\left(\begin{array}{cc}
-\sigma_{y} \hat{k}_{y} & 0 \\
0 & -\sigma_{y} \hat{k}_{y}
\end{array}\right) ;
$$

composed by the Hamiltonians relevant to the inequivalent Dirac points $\vec{K}^{(+)}(-\vec{\sigma} \vec{k})$ and $\vec{K}^{(-)}\left(-\vec{\sigma}^{*} \vec{k}\right)\left(\vec{\sigma}=\left(\sigma_{x}, \sigma_{y}\right)\right.$ are the Pauli matrixes) presented originally in Ref. 23]. The matrix I in (11) is the unit matrix and

$$
V(\vec{\rho})=-\frac{\beta}{\sqrt{\left(x-x_{0}\right)^{2}+y^{2}}} ; \quad \beta=\frac{Z e^{2}}{4 \pi \epsilon_{0} \epsilon_{\mathrm{eff}}} ;
$$

is the $2 \mathrm{D}$ Coulomb impurity potential, $\epsilon_{\mathrm{eff}}$ is the effective dielectric constant related to the static dielectric constant $\epsilon$ of the substrate by [6, 25]

$$
\epsilon_{\mathrm{eff}}=\frac{1}{2}\left(1+\epsilon+\pi q_{0}\right) ; q_{0}=\frac{e^{2}}{4 \pi \epsilon_{0} \hbar v_{F}} \simeq 2.2 .
$$

The envelope wave four-vector $\vec{\Psi}$ consists of two vectors $\vec{\Psi}_{A, B}$ describing the motion of the electron in sublattices $A$ and $B$ of graphene

$$
\vec{\Psi}(\vec{\rho})=\vec{\Psi}_{A}(\vec{\rho})+\vec{\Psi}_{B}(\vec{\rho}) ;
$$

each determined by the wave functions $\psi_{A, B}^{(+,-)}$

$$
\vec{\Psi}_{A}=\left\{\begin{array}{c}
\psi_{A}^{(+)} \\
0 \\
\psi_{A}^{(-)} \\
0
\end{array}\right\} ; \quad \vec{\Psi}_{B}=\left\{\begin{array}{c}
0 \\
\psi_{B}^{(+)} \\
0 \\
\psi_{B}^{(-)}
\end{array}\right\}
$$

The total $A, B$ state implies the multiplication of the $\psi_{A, B}^{(+,-)}$functions with the factors $\exp \left\{\mathrm{i} \vec{K}^{(+,-)} \vec{\rho}\right\}$, respectively. The boundary conditions for the armchair ribbon require the total wave function to vanish at both edges $x= \pm d / 2$ for both $A, B$ superlattices [1] 


$$
e^{\mathrm{i} K x} \psi_{A, B}^{(+)}(\vec{\rho})+e^{-\mathrm{i} K x} \psi_{A, B}^{(-)}(\vec{\rho})=0 \text { at } x= \pm \frac{d}{2},
$$

where $\vec{K}^{(+,-)}=( \pm K, 0) ; K=4 \pi / 3 a_{0}, a_{0}=2.46 \AA$ is the graphene superlattice constant.

The basis wave vectors $\vec{\Phi}_{N}(x)$ and the energies $\varepsilon_{N}$ describing the transverse size-quantized $x$-states are derived from equation

$$
\hat{\mathrm{H}}_{0}\left(\hat{k}_{x}\right) \vec{\Phi}_{N}(x)=\varepsilon_{N} \vec{\Phi}(x)
$$

to obtain

$\vec{\Phi}_{N}(x)=\frac{1}{\sqrt{2}}\left[\vec{\Phi}_{N A}(x)+\vec{\Phi}_{N B}(x)\right] ; \vec{\Phi}_{N A}(x)=\left\{\begin{array}{c}\varphi_{N A}^{(+)} \\ 0 \\ \varphi_{N A}^{(-)} \\ 0\end{array}\right\} ; \vec{\Phi}_{N B}=\left\{\begin{array}{c}0 \\ \varphi_{N B}^{(+)} \\ 0 \\ \varphi_{N B}^{(-)}\end{array}\right\}$

, where

$$
-\varphi_{N A}^{(+)}=\varphi_{N A}^{(-) *}=\varphi_{N B}^{(+)}=-\varphi_{N B}^{(-) *}=\varphi_{N 0}
$$

with

$$
\begin{aligned}
& \varphi_{N 0}(x)=\frac{1}{\sqrt{2 d}} \exp \left\{\mathrm{i}\left[x \frac{\pi}{d}(N-\tilde{\sigma})-\frac{\pi}{2}\left(N+\left[\frac{K d}{\pi}\right]\right)\right]\right\} \\
& \varepsilon_{N}=|N-\tilde{\sigma}| \frac{\pi p}{d} ; N=0, \pm 1, \pm 2, \ldots ; \quad \tilde{\sigma}=\frac{K d}{\pi}-\left[\frac{K d}{\pi}\right]
\end{aligned}
$$

We consider transverse states with positive energies $\varepsilon_{N}>0$ in the armchair ribbon of width $d$ providing the gaped (insulator) structure $\tilde{\sigma} \neq 0$ [1]. It follows from eq.(7) that the energy levels $\varepsilon_{N}$ as a function of width $d$ are the oscillations describing by parameter $\tilde{\sigma}(d)$ imposed on the decreasing curve $\sim d^{-1}$. Below we ignore these oscillations keeping $\tilde{\sigma}=$ const.

The boundary conditions (44) after substitution of $\psi_{A, B}^{(+,-)}$by $\varphi_{N A, B}^{(+,-)}$, respectively, are satisfied. The wave vectors $\vec{\Phi}_{N A, B}$ form orthonormal subsets, for which

$$
\begin{aligned}
\hat{\mathrm{H}}_{0}\left(\hat{k}_{x}\right) \vec{\Phi}_{N A(B)} & =\varepsilon_{N} \vec{\Phi}_{N B(A)} ;\left\langle\vec{\Phi}_{N^{\prime} A(B)} \mid \vec{\Phi}_{N B(A)}\right\rangle=0 ; \\
\left\langle\vec{\Phi}_{N^{\prime} A(B)} \mid \vec{\Phi}_{N A(B)}\right\rangle & =\delta_{N^{\prime} N} ; \quad\left\langle\vec{\Phi}_{N^{\prime}} \mid \vec{\Phi}_{N}\right\rangle=\delta_{N^{\prime} N} .
\end{aligned}
$$

The boundary conditions (4) imposed on the wave vector $\vec{\Psi}(\vec{\rho})$ (3) force us to expand the wave vectors $\vec{\Psi}_{A, B}$ in series 


$$
\vec{\Psi}_{A, B}(\vec{\rho})=\Sigma_{N} u_{N A, B}(y) \vec{\Phi}_{N A, B}(x) ; \text { i.e. } \psi_{A, B}^{(+,-)}(\vec{\rho})=\Sigma_{N} u_{N A, B}(y) \varphi_{N A, B}^{(+,-)}(x)
$$

with respect to the basis functions $\varphi_{N A, B}^{(+,-)}(x)$ taking for the coefficients $u_{N A, B}^{(+)}(y)=$ $u_{N A, B}^{(-)}(y) \equiv u_{N A, B}(y)$. Substituting the wave vector $\vec{\Psi}$ (3) with the wave vectors $\vec{\Psi}_{A, B}$ and the wave functions $\psi_{A, B}^{(+,-)}(\vec{\rho})$ into eq. (1) and subsequently using the properties (8) we obtain by the standard method the set of equations for the wave functions

$$
\left.\begin{array}{c}
v_{N}^{(1)}=\frac{1}{\sqrt{2}}\left(u_{N B}+u_{N A}\right) ; \quad v_{N}^{(2)}=\frac{1}{\sqrt{2}}\left(u_{N B}-u_{N A}\right) ; \\
\frac{d v_{N}^{(1)}(y)}{d y}-\frac{1}{p}\left(E+\varepsilon_{N}-V_{N N}(y)\right) v_{N}^{(2)}(y)+\frac{1}{p} \sum_{N^{\prime} \neq N} V_{N^{\prime} N}(y) v_{N^{\prime}}^{(2)}(y)=0 ; \\
\frac{d v_{N}^{(2)}(y)}{d y}+\frac{1}{p}\left(E-\varepsilon_{N}-V_{N N}(y)\right) v_{N}^{(1)}(y)-\frac{1}{p} \sum_{N^{\prime} \neq N} V_{N^{\prime} N}(y) v_{N^{\prime}}^{(1)}(y)=0 ;
\end{array}\right\}
$$

where the potential $V(\vec{\rho})$ is given by eq. (2). At $|y| \gg d$

$$
V_{N^{\prime} N}(y)=-\frac{\beta}{|y|}\left[\delta_{N^{\prime} N}+O\left(\frac{d^{2}}{y^{2}}\right) \delta_{\left|N^{\prime}-N\right|(2 s+1)}\right] ; s=0,1,2, \ldots
$$

As expected in the limiting case $d \rightarrow 0$ eqs. (10) decompose into the sets describing the 1D Coulomb states, while in the absence of the impurity centre $\left(V_{N^{\prime} N}=0\right)$ we arrive at the wave functions $u_{N A, B} \sim \exp \left( \pm \mathrm{i} k_{y} y\right)$ and the energies $E_{N}^{2}\left(k_{y}\right)=\varepsilon_{N}^{2}+p^{2} k_{y}^{2}$ of free electrons in the armchair nanoribbon [23, 1.

Below we solve the set (10) in the adiabatic approximation implying the longitudinal $y$-motion governed by the quasi-Coulomb potentials $V_{N^{\prime} N}(y)$ to be much slower than the transverse $x$-motion affected by the boundaries of the narrow ribbon. The Coulomb potential (2) is assumed to be small compared to the ribbon confinement. In the case of $\tilde{\sigma}<0.5$ the lowest three sub-bands are specified by indices $N=0,1,-1$. The set (10) corresponding to these subbands becomes

$$
\begin{gathered}
v_{0}^{(1)^{\prime}}-\frac{1}{p}\left(E+\varepsilon_{0}-V_{00}\right) v_{0}^{(2)}+\frac{1}{p}\left[V_{10} v_{1}^{(2)}+V_{-10} v_{-1}^{(2)}\right]=0 ; \\
v_{0}^{(2)^{\prime}}+\frac{1}{p}\left(E-\varepsilon_{0}-V_{00}\right) v_{0}^{(1)}-\frac{1}{p}\left[V_{10} v_{1}^{(1)}+V_{-10} v_{-1}^{(1)}\right]=0 ; \\
v_{1}^{(1)^{\prime}}-\frac{1}{p}\left(E+\varepsilon_{1}-V_{11}\right) v_{1}^{(2)}+\frac{1}{p}\left[V_{01} v_{0}^{(2)}+V_{-11} v_{-1}^{(2)}\right]=0 ; \\
v_{1}^{(2)^{\prime}}+\frac{1}{p}\left(E-\varepsilon_{1}-V_{11}\right) v_{1}^{(1)}-\frac{1}{p}\left[V_{01} v_{0}^{(1)}+V_{-11} v_{-1}^{(1)}\right]=0 ; \\
v_{-1}^{(1)^{\prime}}-\frac{1}{p}\left(E+\varepsilon_{-1}-V_{-1-1}\right) v_{-1}^{(2)}+\frac{1}{p}\left[V_{0-1} v_{0}^{(2)}+V_{1-1} v_{1}^{(2)}\right]=0 ; \\
v_{-1}^{(2)^{\prime}}+\frac{1}{p}\left(E-\varepsilon_{-1}-V_{-1-1}\right) v_{-1}^{(1)}-\frac{1}{p}\left[V_{0-1} v_{0}^{(1)}+V_{1-1} v_{1}^{(1)}\right]=0 ;
\end{gathered}
$$




\section{Single-subband approximation}

At the first stage we neglect the coupling between the states corresponding to the subbands with different $N$. The reason for this is that in the narrow ribbon of small width $d$ the diagonal potentials $V_{N N}$ dominate the off-diagonal terms $V_{N^{\prime} N}\left(N^{\prime} \neq N\right)$ almost everywhere but for a small region $|y| \leq d$ (see eq. (12)). In this case $V_{N^{\prime} N}=V_{N N} \delta_{N^{\prime} N}$ and the set (10) decomposes into independent subsets each specified by an index $N$. The 1D impurity states are then governed by the potential

$$
\begin{gathered}
V_{N N}(y)=\frac{\beta}{d} \ln \frac{\frac{4 y^{2}}{d_{1} d_{2}}}{\left(1+\sqrt{1+\frac{4 y^{2}}{d_{1}^{2}}}\right)\left(1+\sqrt{1+\frac{4 y^{2}}{d_{2}^{2}}}\right)}=\left\{\begin{array}{cc}
\frac{\beta}{d} \ln \frac{y^{2}}{d_{1} d_{2}} ; & \frac{|y|}{d_{1,2}} \ll 1 \\
-\frac{\beta}{|y|} ; & \frac{|y|}{d_{1,2}} \gg 1
\end{array}\right. \\
d_{1,2}=d \pm 2 x_{0} ; \quad-\frac{d}{2}<x_{0}<+\frac{d}{2} ;
\end{gathered}
$$

The set (10) for $y>0$ with $V_{N^{\prime} N}=0$ is solved by matching in the interme-

diate region the corresponding solutions $\left\{v_{N}^{(1)}, v_{N}^{(2)}\right\}$ one of which is valid in the inner region close to the impurity centre and the other represents a solution of the outer region distant from the centre. This method was originally developed by Hasegava and Howard [26] in studies of excitons subject to strong magnetic fields and then successfully employed for the investigation of the impurity and exciton states in quantum wells [27, super-lattices [28] and quantum wires [18].

\subsection{Inner region}

In the inner region

$0 \leq y \ll r_{0}\left(r_{0}=p\left|\varepsilon_{N}^{2}-E^{2}\right|^{-1 / 2}\right.$ is the effective size of the Coulomb state) an iteration procedure is performed. The first integration of the set (10), in which we neglect the terms consisting of the energies $\varepsilon_{N}$ and $E$, with the trial functions $v_{N 0}^{(1)}=a_{N}^{(1)} ; v_{N 0}^{(2)}=a_{N}^{(2)}$ gives

$$
v_{N 1}^{(1)}(y)=a_{N}^{(1)}-a_{N}^{(2)} \frac{q}{2 d}\left[d_{1} F\left(f_{1}\right)+d_{2} F\left(f_{2}\right)\right] ; \quad f_{1,2}=\frac{2 y}{d_{1,2}}
$$

where

$$
F(f)=f \ln \frac{\sqrt{1+f^{2}}-1}{f}-\operatorname{arsh} f=\left\{\begin{array}{cc}
f\left(\ln \frac{|f|}{2}-1\right), & |f| \ll 1 \\
-\frac{f}{|f|}(\ln 2|f|+1), & |f| \gg 1
\end{array}\right.
$$

and where

$$
q=\frac{\beta}{p}=\frac{Z e^{2}}{4 \pi \epsilon_{0} \epsilon_{\mathrm{eff}} \hbar v_{F}} ; \quad(q \ll 1)
$$

is the dimensionless strength of the Coulomb potential. 
The function $v_{N 1}^{(2)}(y)$ can be obtained from eq. (15) by replacing $a_{N}^{(1)}$ by $a_{N}^{(2)}$ and $a_{N}^{(2)}$ by $-a_{N}^{(1)}$. Subsequent integration leads to the two independent particular solutions $\left\{v_{N+}^{(1)}, v_{N+}^{(2)}\right\}$ and $\left\{v_{N-}^{(1)}, v_{N-}^{(2)}\right\}$ corresponding to the relationships $a_{N}^{(2)}= \pm \mathrm{i} a_{N}^{(1)}$. The linear combination of these solutions provides the general iteration functions, which read in the region $y \gg d_{1,2}$

$v_{N \text { in }}^{(1)}(y)=R_{N} \sin \left(Q+\zeta_{N}\right) ; v_{N \operatorname{in}}^{(2)}(y)=R_{N} \cos \left(Q+\zeta_{N}\right) ; Q(y)=q \frac{y}{|y|}\left(\ln \frac{4|y|}{D}+1\right)$,

where

$$
D=\sqrt{d_{1} d_{2}} \exp \left\{\frac{1}{4 d}\left(d_{1}-d_{2}\right) \ln \frac{d_{1}}{d_{2}}\right\}
$$

and where $R_{N}$ and $\zeta_{N}$ are the arbitrary magnitude and phase, respectively. Since the potentials (11) satisfy $V_{N N^{\prime}}(y)=V_{N N^{\prime}}(-y)$ the wave two-vectors $\vec{v}_{N}\left\{v_{N}^{(1)}, v_{N}^{(2)}\right\}$ are classified with respect to parity. Further we focus on the even wave vectors for which $\hat{\Pi} \vec{v}_{N}=\vec{v}_{N}$ where $\hat{\Pi}=\hat{\pi} \sigma_{z}$ with $\hat{\pi} v(y)=v(-y)\left(\sigma_{z}\right.$ is the Pauli matrix). The condition of even parity imposed on the wave vector $\vec{v}_{N \text { in }}$ formed by the components (16), implies that the phases $\zeta_{N}$ are equal to the half integer of $\pi$.

Obviously, an alternative way to derive eq. (16) is to solve eqs. (10) for $V_{N N}(y)=-\beta|y|^{-1}, \varepsilon_{N}=E=0$ and then to compare the resulting solutions

$v_{N+,-}^{(1,2)}(y) \sim A y^{ \pm \mathrm{i} q}$, expanded in series up to the terms of the first order of $q$, with those given by eqs. (15). The calculated constant $A$ leads to the functions (16).

\subsection{Outer region}

a) Discrete states

The exact solutions to eqs. (10) at $V_{N^{\prime} N}=0$ for $N \neq N^{\prime}$ in the region $y \gg d_{1,2}$ with $V_{N N}(y)=-\beta y^{-1}$ are calculated by the same method employed in studies of a relativistic electron in hydrogen [29] and super-heavy atoms with the nuclear charge number number $Z>137$ [11, 12, 13, 14.

$$
\left\{\begin{array}{l}
v_{N}^{(1)}(\tau) \\
v_{N}^{(2)}(\tau)
\end{array}\right\}=A_{N}\left\{\begin{array}{c}
\cosh \frac{\psi_{N}}{2} \tau^{-\frac{1}{2}}\left[W_{\kappa, \mu}(\tau)+\frac{\tanh \psi}{q} W_{\kappa+1, \mu}(\tau)\right] \\
\sinh \frac{\psi_{N}}{2} \tau^{-\frac{1}{2}}\left[W_{\kappa, \mu}(\tau)-\frac{\tanh \psi}{q} W_{\kappa+1, \mu}(\tau)\right]
\end{array}\right.
$$

where 


$$
\begin{aligned}
\tau & =\nu_{N} y, \nu_{N}=\frac{2}{p} \sqrt{\varepsilon_{N}^{2}-E^{2}} ; \tanh \psi=\frac{p \nu_{N}}{2 \varepsilon_{N}} ; \kappa=\eta-\frac{1}{2} ; \mu=\text { iq } ; \\
\eta_{N} & =\frac{2 q E}{p \nu_{N}} ; A_{N}^{2}=\frac{\nu_{N}}{2 \Gamma\left(\eta_{N}\right)^{2} \cosh \psi_{N}\left(1+\frac{\eta_{N}^{2}}{q^{2}} \tanh \psi_{N}^{2}\right)}\left(1+\delta_{\eta \eta_{0}}\right) ; \\
\eta_{0} & \ll 1 \text { is the quantum number labeling the ground state }
\end{aligned}
$$

and where $W_{\kappa, \mu}(\tau)$ is the Whittaker function associated with the Kummer function $U$ 30.

$$
W_{\kappa, \mu}(\tau)=e^{-\frac{\tau}{2}} \tau^{\frac{1}{2}+\mu} U(a, c, \tau) ; a=\frac{1}{2}+\mu-\kappa, c=1+2 \mu,
$$

with 31]

$$
U(a, c, \tau)= \begin{cases}\tau^{-a} ; & \tau \gg 1 \\ \frac{\Gamma(1-c)}{\Gamma(a-c+1)}+\frac{\Gamma(c-1)}{\Gamma(a)} \tau^{1-c} ; \operatorname{Re} c=1 ; c \neq 1 ; & \tau \ll 1 .\end{cases}
$$

The functions (18) are normalized to $\int_{-\infty}^{+\infty}\left(v_{N}^{(1) 2}+v_{N}^{(2) 2}\right) d y=1$.

The asymptotic behavior of the outer functions (18) at large distances $y \gg r_{0}$ follows from eqs. (18), (19) and (20)

$$
v_{N}^{(1,2)}(y) \sim \exp \left(-\frac{y}{r_{0}}+\eta_{N} \ln \frac{2 y}{r_{0}}\right) ; r_{0}=\frac{2}{\nu_{N}} .
$$

In the region $\tau \ll 1$ eqs. (18), (19) and (20) lead to the expressions

$$
v_{\text {Nout }}^{(1)}=P_{N} \sin \omega_{N} ; v_{N \text { out }}^{(2)}=P_{N} \cos \omega_{N} ; \omega_{N}(\tau)=q \ln \tau+\Theta_{N},
$$

where

$$
\Theta_{N}=\arg \Gamma(-2 \mathrm{i} q)+\arg \Gamma\left(-\eta_{N}+\mathrm{i} q\right)-\arctan \frac{\eta_{N}}{q}\left(\sqrt{1+\frac{q^{2}}{\eta_{N}^{2}}}-1\right),
$$

and $P_{N}$ are arbitrary constants.

Matching the eqs. (18) and (16) in the overlapping intermediate region $d \ll y \ll r_{0}$ we impose the condition

which yields

$$
\frac{v_{\text {Nin }}^{(1)}(y)}{v_{\text {Nin }}^{(2)}(y)}=\frac{v_{\text {Nout }}^{(1)}(y)}{v_{\text {Nout }}^{(2)}(y)}
$$

$$
\omega_{N}-Q-\zeta_{N}=s \pi ; s=0, \pm 1, \pm 2, \ldots
$$


Using the properties of the arguments of the $\Gamma$-functions in eq. (23) for a small parameter $q \ll 1$ and for the quantum numbers $\eta_{N}=n+\delta_{N n}, n=$ $0,1,2, \ldots, \delta_{N n}<1$ [30] (see also Ref. [11, 13] for details) and choosing $\zeta_{N}=\pi / 2$ we arrive at the equation for the corrections $\delta_{N n}$

$$
\begin{aligned}
\ln q+\frac{1}{q}\left[\arctan \frac{q}{\delta_{N n}}-\right. & \left.\arctan \frac{q}{2\left(n+\delta_{N n}\right)}\right]-\ln \left(n+\delta_{N n}\right) \\
& +\psi(1+n)+\ln \frac{|N-\tilde{\sigma}| \pi D}{2 d}+2 C-1=0,
\end{aligned}
$$

where $n=0,1,2, \ldots, \psi(1+n)$ is the psi-function (logarithmic derivative of the $\Gamma$-function), $C=0.577$ is the Euler constant. The corrections $\delta_{N n}$ calculated from eq. (25) determine the Rydberg series of the discrete energy levels $E_{N n}$ adjacent to the size-quantized energy level $\varepsilon_{N}$

$$
E_{N n}= \begin{cases}\varepsilon_{N}\left[1-\varepsilon_{N} \frac{q^{2}}{2\left(n+\delta_{N n}\right)^{2}}\right] ; & n=1,2, \ldots \\ \frac{\varepsilon_{N}}{\sqrt{1+\frac{q^{2}}{\delta_{N 0}^{2}}}} ; & n=0\end{cases}
$$

which allow to estimate the size of the Coulomb state in eq. (21) $r_{0} \simeq d / \mid N-$ $\tilde{\sigma} \mid \pi q$ for $n=1,2, \ldots$ and

$$
r_{0}=\frac{d}{|N-\tilde{\sigma}| \pi \sqrt{1-\frac{1}{1+\frac{q^{2}}{\delta_{N 0}^{2}}}}} \text { for } n=0 .
$$

Clearly from (26), the existence of the intermediate matching region $d_{1,2} \ll$ $y \ll r_{0}$ is provided for excited state $n=1,2, \ldots$ by the employed above small parameter $q \ll 1$ and for the ground state $n=0$ by the condition

$$
\left(1 \pm \frac{2 x_{0}}{d}\right)|N-\tilde{\sigma}| \pi z_{0} \ll 1 \text {, i.e. } z_{0}=\frac{q}{\delta_{N 0}} \ll 1 .
$$

The correction $\delta_{N 0}=q / z_{0}$ satisfies the transcendental equation

$$
\ln z_{0}+\frac{1}{q}\left[\arctan z_{0}-\arctan \frac{z_{0}}{2}\right]+\ln \frac{|N-\tilde{\sigma}| \pi D}{2 d}+C-1=0,
$$

while the corrections $\delta_{N n}$ for the excited states $n=1,2, \ldots$ can be calculated in an explicit form

$$
\delta_{N n}=q \cot \left\{q\left[-\ln q+\ln n+\frac{1}{2 n}-\psi(1+n)-\ln \frac{|N-\tilde{\sigma}| \pi D}{2 d}-2 C+1\right]\right\}
$$

b) Continuous states

Since our approach to determine the wave function of the continuous states closely resembles that applied above for the wave functions of the discrete states 
only the basic points will be given below. Setting in eqs. (18) $\nu_{N}=-2 \mathrm{i} k$ we obtain

$$
\left\{\begin{array}{l}
v_{N+}^{(1)}(t) \\
v_{N+}^{(2)}(t)
\end{array}\right\}=B_{N}\left\{\begin{aligned}
& \cos \frac{\varphi_{N}}{2} t^{-\frac{1}{2}}\left[W_{\tilde{\kappa}, \mu}(t)-\mathrm{i} \frac{\tan \varphi}{q} W_{\tilde{\kappa}+1, \mu}(t)\right] \\
-\mathrm{i} \quad & \sin \frac{\varphi_{N}}{2} t^{-\frac{1}{2}}\left[W_{\tilde{\kappa}, \mu}(t)+\mathrm{i} \frac{\tan \varphi}{q} W_{\tilde{\kappa}+1, \mu}(t)\right] .
\end{aligned}\right.
$$

where

$$
\begin{aligned}
t & =-2 \mathrm{i} k_{N} y ; k_{N}=\frac{1}{p} \sqrt{E^{2}-\varepsilon_{N}^{2}} ; \tan \varphi=\frac{p k}{\varepsilon_{N}} ; \tilde{\kappa}=\mathrm{i} \frac{q}{\sin \varphi}-\frac{1}{2} ; \\
\mu & =\mathrm{i} q ; B_{N}^{2}=\frac{q^{2}}{2 \pi \tan ^{2} \varphi} \exp \left(-\frac{\pi q}{\sin \varphi}\right) .
\end{aligned}
$$

The wave vectors (29) are normalized to $\delta\left(k-k^{\prime}\right)$.

At large distances $k y \gg 1$ the wave functions (29), (19), (20) have the asymptotic form of the outgoing waves

$$
v_{N+}^{(1,2)}(y) \sim \exp \left\{\mathrm{i} k_{N} y+\mathrm{i} \frac{q}{\sin \varphi} \ln 2 k_{N} y\right\} .
$$

Further we introduce the real wave functions associated with the standing waves

$$
v_{N \text { out }}^{(1,2)}(t)=D_{N}\left[e^{\mathrm{i} \Omega_{N}} v_{N+}^{(1,2)}(t)+e^{-\mathrm{i} \Omega_{N}} v_{N-}^{(1,2)}(t)\right],
$$

where the functions $v_{N-}^{(1,2)}(t)=v_{N+}^{(1,2) *}(t)$ have the asymptotic form of the ingoing waves $\sim \exp \left\{-\mathrm{i} k y-\mathrm{i} \frac{q}{\sin \varphi} \ln 2 k y\right\}$ and $D_{N}$ and $\Omega_{N}$ are the arbitrary magnitude and phase, respectively.

In the region $|t| \ll 1$ eqs. (29), (19) and (20) lead to

$v_{N \text { out }}^{(1)}(t)=D_{N}\left[e^{q \frac{\pi}{2}} \frac{M(\varphi)}{\left|\Gamma_{+}\right|} \cos \left(\Omega_{N}+q \ln |t|+\xi_{+}\right)-e^{-q \frac{\pi}{2}} \frac{M(-\varphi)}{\left|\Gamma_{-}\right|} \cos \left(-\Omega_{N}+q \ln |t|+\xi_{-}\right)\right]$

where

$\Gamma_{+,-}=\Gamma\left[-\mathrm{i} q\left(\frac{1}{\sin \varphi} \pm 1\right)\right] ; \xi_{+,-}=\arg \Gamma(-2 \mathrm{i} q) \mp \arg \Gamma_{+,-} ; M(\varphi)=\frac{\sin \frac{\varphi}{2}+\cos \frac{\varphi}{2}}{1+\sin \varphi}$

The wave functions $v_{N \text { out }}^{(2)}(t)$ can be obtained from eq. (31) by replacing $D_{N}$ by $-D_{N}$ and $\xi_{+,-}$by $\xi_{+,-}-\pi / 2$.

For $q \ll 1, \varphi \ll 1$ the wave functions (31) read

$$
v_{N \text { out }}^{(1)}(t)=D_{N}\left[\sin \chi_{N}-c_{N} \cot \Lambda_{N} \cos \chi_{N}\right] ; v_{N \text { out }}^{(2)}(t)=D_{N}\left[\cos \chi_{N}+c_{N} \cot \Lambda_{N} \sin \chi_{N}\right]
$$


with

$\chi_{N}=q \ln |t|+\frac{1}{2}\left(\xi_{-}+\xi_{+}\right) ; \Lambda_{N}=\Omega_{N}-\frac{1}{2}\left(\xi_{-}-\xi_{+}\right) ; c_{N}=q \frac{\pi}{2}\left(1+\operatorname{coth} \frac{q \pi}{\varphi}\right)$.

Similar to the case of the discrete states we obtain the equation for the phase $\Lambda_{N}$ on equating the ratios $v_{N}^{(1)}(y) / v_{N}^{(2)}(y)$ taken for the iteration (16) and outer functions (32)

$$
\cot \Lambda_{N}=\frac{1}{c_{N}} \tan \left(\chi-Q-\zeta_{N}\right)
$$

Since 30

$$
\frac{1}{2}\left(\xi_{-}-\xi_{+}\right)=\frac{\pi}{2}-\Omega^{(0)} ; \quad \Omega^{(0)}=\sum_{j=1}^{\infty}\left(\frac{q}{j \varphi}-\arctan \frac{q}{j \varphi}\right)
$$

eq. (34) acquires for $\zeta_{N}=\pi / 2$ an explicit form

$$
\cot \Omega_{N}=\frac{\frac{\pi}{2}\left(1+\operatorname{coth} \frac{q \pi}{\varphi}\right)}{\ln \frac{2}{k D}-\frac{1}{2}\left[\psi\left(1+\mathrm{i} \frac{q}{\sin \varphi}+\right)+\psi\left(1-\mathrm{i} \frac{q}{\sin \varphi}+\right)\right]-2 C+1} .
$$

Since at $q \ll 1$ the phase $\Omega^{(0)}=\frac{1}{3} \zeta(3) \frac{q^{3}}{\varphi^{3}}(\zeta(s)$ is the Riemann zeta function with $\zeta(3)=1.20)$ is the value of the higher order of smallness $\sim q^{3} \ll 1$ we set in eq. (35) $\Omega^{(0)}=0$.

As expected, setting in the functions $v_{\text {Nout }}^{(1,2)} k_{N}=\frac{\mathrm{i} \nu_{N}}{2},\left(\Gamma_{+}=\Gamma_{-}^{*}\right)$ and then matching these functions with the iteration functions $v_{N i t}^{(1,2)}(16)$ we obtain the equation for the phase $\Omega_{N}$. Substituting this result into equation $\cot \Omega_{N}=\mathrm{i}$ determining the poles of the $S$ matrix with $S=\exp \left(2 \mathrm{i} \Omega_{N}\right)$ [10, 29] we arrive at eqs. (24), (25) for the discrete energy levels.

\section{Double-subband approximation}

Below we consider the coupling between the ground $N=0$ and first excited $N=$ 1 states described by the system of the four upper eqs. (13) at $V_{-10}=V_{-11}=0$. Applying the iteration method with the trial functions $v_{0}^{(1,2)}=a_{0}^{(1,2)}$ and $v_{1}^{(1,2)}=$ $a_{1}^{(1,2)}$ we arrive at two particular linear independent four-vectors, having the components $v_{0,1}^{(1,2)}$ calculated for $a_{0}^{(2)}=\mathrm{i} a_{0}^{(1)}, a_{1}^{(2)}=\mathrm{i} a_{1}^{(1)}$ and $a_{0}^{(2)}=-\mathrm{i} a_{0}^{(1)}, a_{1}^{(2)}=$ $-\mathrm{i} a_{1}^{(1)}$. The linear combination of these vectors taken for $a_{0,1}^{(2)}=R_{0,1} \exp \left[\mathrm{i}\left(\zeta_{0,1}-\frac{\pi}{2}\right)\right]$ provides the general expression for the iteration four-vector with the components

$$
\left.\begin{array}{c}
v_{0 i t}^{(1)}(y)=R_{0} \sin \left(Q+\zeta_{0}\right)+R_{1} q \gamma_{01} \cos \zeta_{1} ; \\
v_{0 i t}^{(2)}(y)=R_{0} \cos \left(Q+\zeta_{0}\right)-R_{1} q \gamma_{01} \sin \zeta_{1} ;
\end{array}\right\}
$$


where $R_{0,1}$ and $\zeta_{0,1}$ are an arbitrary constant and phase, respectively. The parameter

$$
\begin{aligned}
\pi \gamma_{0,1} & =\cos \alpha_{0}\left[\mathrm{Ci}\left(\frac{\pi}{2}+\alpha_{0}\right)-\operatorname{Ci}\left(\frac{\pi}{2}-\alpha_{0}\right)\right] \\
& +\sin \alpha_{0}\left[\operatorname{Si}\left(\frac{\pi}{2}+\alpha_{0}\right)+\operatorname{Si}\left(\frac{\pi}{2}-\alpha_{0}\right)\right], \alpha_{0}=\frac{\pi x_{0}}{d} .
\end{aligned}
$$

consisting of the integral sine $\mathrm{Si}$ and cosine $\mathrm{Ci}$ [30], describes the coupling induced by the potentials $V_{01}=V_{10}$ (11). The functions $v_{1 \text { in }}^{(1)}(y)$ and $v_{1 \text { in }}^{(2)}(y)$ can be obtained from the functions $v_{\text {oin }}^{(1)}(y)$ and $v_{\text {oin }}^{(2)}(y)$ (36) by mutual replacing $R_{0} \leftrightarrow R_{1}$.

Equating the ratios of the functions of the continuous spectrum (32) $v_{\text {oout }}^{(1)}(t) / v_{\text {oout }}^{(2)}(t)$ and the iteration functions (36) $v_{\text {oin }}^{(1)}(y) / v_{\text {oin }}^{(2)}(y)$ and then matching the ratios of the functions of the discrete states (22) $v_{1 \text { out }}^{(1)}(\tau) / v_{1 \text { out }}^{(2)}(\tau)$ and the iteration functions $v_{1 \text { in }}^{(1)}(y) / v_{1 \text { in }}^{(2)}(y)$ we obtain the set of equations

$$
\left.\begin{array}{r}
R_{0}\left[\sin \left(\chi_{0}-Q-\zeta_{0}\right)-c_{0} \cot \Lambda_{0} \cos \left(\chi_{0}-Q-\zeta_{0}\right)\right]+ \\
R_{1} q \gamma_{01}\left[\cos \left(\chi_{0}-\zeta_{1}\right)-c_{0} \cot \Lambda_{0} \sin \left(\chi_{0}-\zeta_{1}\right)\right]=0 ; \\
R_{0} q \gamma_{01} \cos \left(\omega_{1}-\zeta_{0}\right)-R_{1} \sin \left(\omega_{1}-Q-\zeta_{1}\right)=0,
\end{array}\right\}
$$

where the functions $\chi_{N}, Q, \omega_{N}$ are defined by eqs. (33), (16), (22), respectively. In the limiting case of negligible coupling the set (38) decomposes into two independent equations relevant to the discrete (24), (25) and continuous (34) states.

Solving the set (38) by the determinantal method we obtain the equation for $\cot \Lambda_{0}$, which is then expanded in series with respect to the parameter $q \ll 1$. Keeping at $\zeta_{N}=\pi / 2$ the terms of the first order $\sim q$ we arrive at the equation for the phase $\Omega_{0}$ in an explicit form

$$
\cot \Omega_{0}=\frac{c_{0}\left(\omega_{1}-Q-\frac{\pi}{2}\right)}{\left(\omega_{1}-Q-\frac{\pi}{2}\right)\left(Q-\chi_{0}+\frac{\pi}{2}\right)-q^{2} \gamma_{0,1}^{2}} .
$$

Substituting eq. (39) into equation [10, 29, 32.

$$
\cot \Omega_{0}=\mathrm{i}
$$

the complex quantum numbers $\eta_{1}$ introduced in eq. (18) can be calculated, which in turn determine the complex energy levels $E_{1 n}$ adjacent to the sizequantized first excited energy level $\varepsilon_{1}=(1-\tilde{\sigma}) \pi p / d$

$$
E_{1 n}=\varepsilon_{1}-\varepsilon_{1} \frac{q^{2}}{2\left(n+\delta_{1 n}^{2}\right)}+W_{1 n}-\mathrm{i} \frac{\Gamma_{1 n}}{2} ; n=0,1,2 \ldots,
$$

where the second term in the right-hand part is the Rydberg series of the energy levels associated with the quasi-Coulomb diagonal potential $V_{11}(y)$ (14) (no 
coupling). The following notation in eq. (41) for the resonant shift $W_{1 n}$ and resonant width $\Gamma_{1 n}$ both induced by the inter-subband $N=0,1$ interaction is used

$$
\Gamma_{1 n}=2 \varepsilon_{1} \frac{q^{2}}{\left(n+\delta_{1 n}\right)^{3}} G_{n}\left(\delta_{1 n}\right) q \gamma_{0,1}^{2} B_{0,1}
$$

and

$$
W_{1 n}=-\varepsilon_{1} \frac{q^{2}}{\left(n+\delta_{1 n}\right)^{3}} G_{n}\left(\delta_{1 n}\right) q^{2} \gamma_{0,1}^{2} A_{0,1} .
$$

In eqs. (42) and (43)

$$
\begin{gathered}
A_{0,1}=B_{0,1}^{2}\left(\ln \frac{k_{0} d}{2}+\ln \frac{D}{d}+C-1\right) ; \quad B_{0,1}=\frac{2 \varepsilon_{0}}{p k_{0}} . \\
G_{0}^{-1}\left(\delta_{10}\right)=\delta_{10}^{-1}+\left(\delta_{10}^{2}+q^{2}\right)^{-1}-\left(2 \delta_{10}^{2}+\frac{1}{2} q^{2}\right)^{-1}, G_{n}\left(\delta_{1 n}\right)=\delta_{1 n}^{2}+q^{2}, n=1,2, \ldots,
\end{gathered}
$$

where the corrections $\delta_{1 n}$ can be calculated from eqs. (25) (27) (28). In the logarithmic approximation $q \ln q^{-1} \ll 1, G_{0}\left(\delta_{10}\right)=2 \delta_{10}^{2}, \quad G_{n}\left(\delta_{1 n}\right)=\delta_{1 n}^{2}, n=$ $1,2, \ldots$ The quantum number $k_{0}$ can be found from equation

$$
E^{2}=\varepsilon_{0}^{2}+p^{2} k_{0}^{2}=\varepsilon_{1}^{2}\left[1-\frac{q^{2}}{\left(n+\delta_{1 n}\right)^{2}}\right]
$$

with (7) for $\varepsilon_{0,1}$.

In conclusion of this section note that the equation absolutely identical to eqs. (41) - (43) can be derived by matching the real iteration functions $v_{1 \text { in }}^{(1,2)}(y)$ (16) and complex functions of the continuous states $v_{0+}^{(1,2)}(t)$ (29) having the asymptotic form of the outgoing wave.

\section{Three-subband approximation}

In this section we consider the coupling between the discrete states adjacent to the highest size-quantized level $\varepsilon_{-1}$ and and the continuous states attributed to the low-lying levels $\varepsilon_{0}$ and $\varepsilon_{1}$. Below we neglect in the set (13) the offdiagonal potentials $V_{01}$ and $V_{10}$ describing the interactions of the $N=0,1$ subbands. Extending the iteration procedure employed above for the singleand double- subband approximation to the present stage with the trial functions $v_{N}^{(1,2)}=a_{N 1,2}$ we arrive at two particular linear independent sixfold vectors $\vec{V}_{+,-}\left(v_{0}^{(1)}, v_{0}^{(2)}, v_{1}^{(1)}, v_{1}^{(2)}, v_{-1}^{(1)}, v_{-1}^{(2)}\right)$ calculated for $a_{N+,-}^{(2)}= \pm \mathrm{i} a_{N+,-}^{(1)}, N=$ $0,1,-1$. Taking $a_{N+,-}^{(2)}=$

$R_{N} \exp \left[ \pm \mathrm{i}\left(\zeta_{N}-\frac{\pi}{2}\right)\right]$, we obtain the components $v_{N}^{(1,2)}$ of the total iteration sixfold vector $\vec{V}_{\text {in }}=\vec{V}_{+}+\vec{V}_{-}$ 


$$
\left.\begin{array}{l}
v_{0 \text { in }}^{(1)}(y)=R_{0} \sin \left(Q+\zeta_{0}\right)+R_{-1} q \gamma_{0,-1} \cos \zeta_{-1} ; \\
v_{1 i n}^{(1)}(y)=R_{1} \sin \left(Q+\zeta_{1}\right)+R_{1} q \gamma_{1,-1} \cos \zeta_{-1} ; \\
v_{-1 \text { in }}^{(1)}(y)=R_{-1} \sin \left(Q+\zeta_{-1}\right)+R_{0} q \gamma_{0,-1} \cos \zeta_{0}+R_{1} q \gamma_{1,-1} \cos \zeta_{1},
\end{array}\right\}
$$

where $Q(y)$ is determined in eq. (16) and $R_{N}$ and $\zeta_{N}$ are arbitrary constants and phases, respectively. The parameter $\gamma_{0,-1}=\gamma_{0,1}$ (37), while

$$
\begin{array}{r}
2 \pi \gamma_{1,-1}=\cos 2 \alpha_{0}\left[-\operatorname{Si}\left(\pi+2 \alpha_{0}\right)-\operatorname{Si}\left(\pi-2 \alpha_{0}\right)\right] \\
+\sin 2 \alpha_{0}\left[\operatorname{Ci}\left(\pi+2 \alpha_{0}\right)-\operatorname{Ci}\left(\pi-2 \alpha_{0}\right)\right], \alpha_{0}=\frac{\pi x_{0}}{d}
\end{array}
$$

describes the coupling induced by the potentials $V_{-11}=V_{1-1}$ (11). The functions $v_{N \text { it }}^{(2)}(y)$ can be obtained from the functions $v_{N \text { it }}^{(1)}(y)$ (44), respectively, by replacing $\sin \left(Q+\zeta_{N}\right)$ by $\cos \left(Q+\zeta_{N}\right), \cos \left(\zeta_{N}\right)$ by $\sin \left(\zeta_{N}\right)$ and $q$ by $-q$.

As mentioned above further we match the wave functions of the continuous spectrum $v_{N}^{(1,2)}(y), N=0,1$ having the asymptotic form of the outgoing wave $v_{N+}^{(1,2)}(29)$ to give in turn the sixfold vector $\vec{V}_{\text {out }}$ with the components

$$
\begin{gathered}
v_{N \text { out }}^{(1)}(y)=\left(1+c_{N}\right) \exp \left[\mathrm{i}\left(q \ln 2 k_{N} y+\xi_{+}\right)\right] \\
-\left(1-c_{N}\right) \exp \left[-\imath\left(q \ln 2 k_{N} y+\xi_{-}\right)\right], N=0,1,
\end{gathered}
$$

where $c_{N}$ and $\xi_{+,-}$are given in eqs. (32) (31) and (29). The wave functions $v_{\text {Nout }}^{(2)}(y), N=0,1$ can be obtained from the functions $v_{\text {Nout }}^{(1)}(y)$ (46), respectively, by replacing $\xi_{+,-}$by $\xi_{+,-}+\frac{\pi}{2}$. The wave functions $v_{-1 \text { out }}^{(1,2)}(y)$ have the form (22), in which $\nu_{N}$ (18) and $\Theta_{N}$ (23) are calculated for $N=-1$.

Matching the sixfold wave vectors $\vec{V}_{\text {in }}$ and $\vec{V}_{\text {out }}$ within the intermediate region by imposing the conditions

$$
\frac{v_{\text {Nout }}^{(1)}}{v_{\text {Nout }}^{(2)}}=\frac{v_{\text {Nin }}^{(1)}}{v_{N \text { in }}^{(2)}}, \quad N=0,1,-1,
$$

where $v_{N \text { out }}^{(1,2)}$ are given by eqs. (46) and (18) and $v_{N \text { in }}^{(1,2)}$ by eqs. (44) we obtain

$$
\left.\begin{array}{l}
R_{0}\left[A_{0}^{(-)} \cos \left(Q+\zeta_{0}\right)-\mathrm{i} A_{0}^{(+)} \sin \left(Q+\zeta_{0}\right)\right]-R_{-1} q \gamma_{0,-1}\left[A_{0}^{(-)} \sin \zeta_{-1}+\mathrm{i} A_{0}^{(+)} \cos \zeta_{-1}\right]=0 ; \\
R_{1}\left[A_{1}^{(-)} \cos \left(Q+\zeta_{1}\right)-\mathrm{i} A_{1}^{(+)} \sin \left(Q+\zeta_{1}\right)\right]-R_{-1} q \gamma_{1,-1}\left[A_{1}^{(-)} \sin \zeta_{-1}+\mathrm{i} A_{1}^{(+)} \cos \zeta_{-1}\right]=0 ; \\
R_{-1} \sin \left(\omega_{-1}-Q-\zeta_{-1}\right)-R_{1} q \gamma_{1,-1} \cos \left(\omega_{-1}-Q-\zeta_{1}\right)-R_{0} q \gamma_{0,-1} \cos \left(\omega_{-1}-Q-\zeta_{0}\right)=0,
\end{array}\right\}
$$

In eqs. (47)

$$
A_{N}^{(+,-)}=\left(1+c_{N}\right) \exp \left[\mathrm{i}\left(q \ln 2 k_{N} y+\xi_{+}\right)\right] \pm\left(1-c_{N}\right) \exp \left[-\mathrm{i}\left(q \ln 2 k_{N} y+\xi_{-}\right)\right]
$$


and $Q(y)$ and $\omega_{-1}(y)$ are introduced by eqs. (16) and (22) for $N=-1$, respectively.

Solving the set (47) by the determinantal method we obtain the equations for the complex quantum numbers $\eta_{N}=2 q E / p \nu_{-1}$ (18), in which we take for the phases $\zeta_{N}=\frac{\pi}{2}, N=0,1,-1$ and keep the terms of the first order of $q \ll 1$

$$
\omega_{-1}-Q-\frac{\pi}{2}=q^{2} \sum_{N=0,1} \gamma_{N,-1}\left(q A_{N,-1}+\mathrm{iB}_{\mathrm{N},-1}\right)
$$

with

$$
A_{N,-1}=B_{N,-1}^{2}\left(\ln \frac{k_{N} d}{2}+\ln \frac{D}{d}+C-1\right) ; \quad B_{N,-1}=\frac{2 \varepsilon_{N}}{p k_{N}} .
$$

and the quantum numbers $k_{0,1}$ are obtained from

$$
E^{2}=\varepsilon_{0}^{2}+p^{2} k_{0}^{2}=\varepsilon_{1}^{2}+p^{2} k_{1}^{2}=\varepsilon_{-1}^{2}\left[1-\frac{q^{2}}{\left(n+\delta_{-1 n}\right)^{2}}\right]
$$

with (7) for $\varepsilon_{-1}$.

The complex quantum numbers $\eta$ calculated from eq. (48) lead to the complex impurity energy levels adjacent to the size-quantized second excited subband $\varepsilon_{-1}$

$$
E_{-1 n}=\varepsilon_{-1}-\varepsilon_{-1} \frac{q^{2}}{2\left(n+\delta_{-1 n}\right)^{2}}+W_{-1 n}-\imath \frac{\Gamma_{-1 n}}{2}, \quad n=0,1,2, \ldots
$$

where the resonant width $\Gamma_{-1 n}$ and shift $W_{-1 n}$ have the form

$$
\Gamma_{-1 n}=2 \varepsilon_{-1} \frac{q^{2}}{\left(n+\delta_{-1 n}\right)^{3}} G_{n} q\left(\gamma_{1,-1}^{2} B_{1,-1}+\gamma_{0,-1}^{2} B_{0,-1}\right)
$$

and

$$
W_{-1 n}=-\varepsilon_{-1} \frac{q^{2}}{\left(n+\delta_{-1 n}\right)^{3}} G_{n} q^{2}\left(\gamma_{1,-1}^{2} A_{1,-1}+\gamma_{0,-1}^{2} A_{0,-1}\right) .
$$

The coefficients $G_{n}$ are defined in eqs. (42) and (43).

\section{Discussion}

We define the binding energy of the electron $E_{N n}^{(b)}$ in the $n$-th quasi-Coulomb state associated with the $N$ size-quantized subband as the real part of the difference between the size-quantized energy $\varepsilon_{N}$ (7) of the free electron and the energy of the impurity electron $E_{N n}$ given by eqs. (26), (41) and (41) for the ground $N=0$, first $N=1$ and second $N=-1$ excited subbands, respectively. Since the resonant shifts $W_{N n}$ (43), (51) are of the order of $q^{2} \ll 1\left(W_{0 n}=0\right)$ with respect to the Rydberg energies determined by the second terms in the right-hand parts of eqs. (26), (41) and (41) the binding energies read 


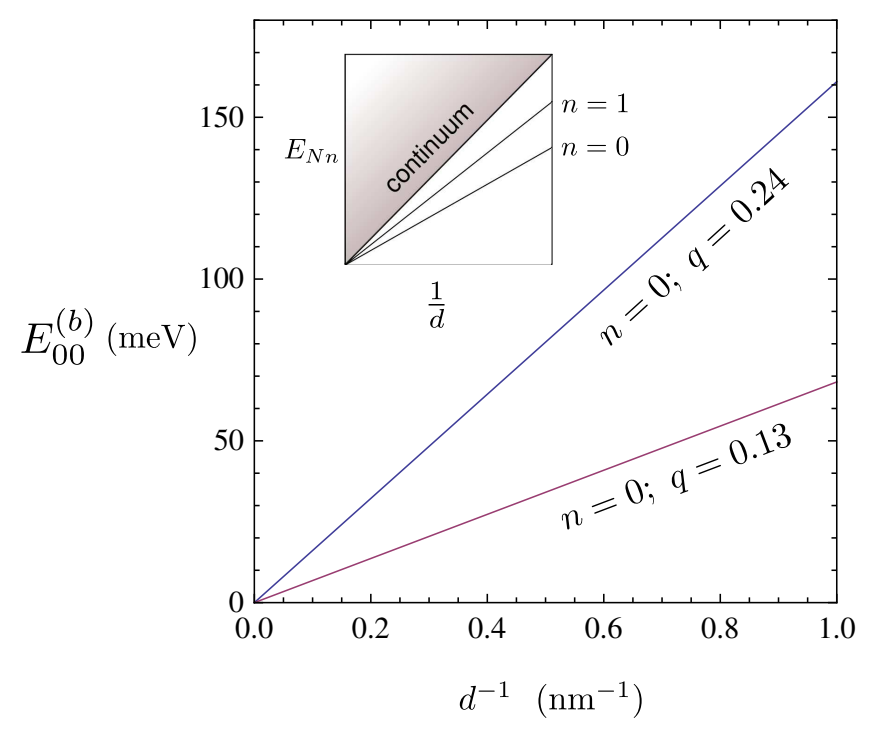

Figure 2: The binding energy $E_{0 n}^{(b)}(\underline{52})$ of the ground state $n=0$ calculated for $(q=0.13 ; 0.24)$ as a function of the reciprocal width $\frac{1}{d}$ of the GNR. Impurity is placed symmetrically to the boundaries $\left(x_{0}=0\right)$. The parameter $\tilde{\sigma}=0.3$.

$$
E_{N n}= \begin{cases}\varepsilon_{N} \frac{q^{2}}{2\left(n+\delta_{N n}\right)^{2}} ; & n=1,2, \ldots \\ \varepsilon_{N}\left[1-\frac{1}{\sqrt{1+\frac{q^{2}}{\delta_{N 0}^{2}}}}\right] ; & n=0\end{cases}
$$

where the corrections $\delta_{N n}$ can be calculated in the single-subband approximation from eqs. (27) and (28) for the ground $n=0$ and excited $n=1,2, \ldots$ impurity states, respectively. It follows from eqs. (52) and (7) that the binding energy $E_{N n}^{(b)} \sim \varepsilon_{N} \sim d^{-1}$ and the oscillatory part of $\varepsilon_{N}$ (see $\tilde{\sigma}(d)$ in eq. (77)) decrease with increasing the ribbon width $d$. In an effort to render our calculations close to an experimental setup, we take below for the estimates of the expected values the parameters $q=0.13(\epsilon \simeq 25)[33$ and $q=0.24(\epsilon \simeq 10)$ corresponding to the $\mathrm{HfO}_{2}$ and sapphire, respectively, employed as substrates for GNR 34. The latter parameter $q$ is close to the limit caused by the condition $z_{0} \ll 1$ (see below eq. (26) ). Further we focus on the monotonic dependence $\sim d^{-1}$ and keep the the parameter $\tilde{\sigma}$ in eq. (77) for the levels $\varepsilon_{N}$ to be $\tilde{\sigma} \simeq 0.3$.

The dependencies of the binding energies on the width of the GNR $d$ for the ground state for different strengths of the impurity potential are given in Fig. 2. These graphs, while ignoring the oscillations, are qualitatively completely in line with the data of the numerical calculations and experimental observations recently performed with the related Coulomb systems. The exciton effects in 


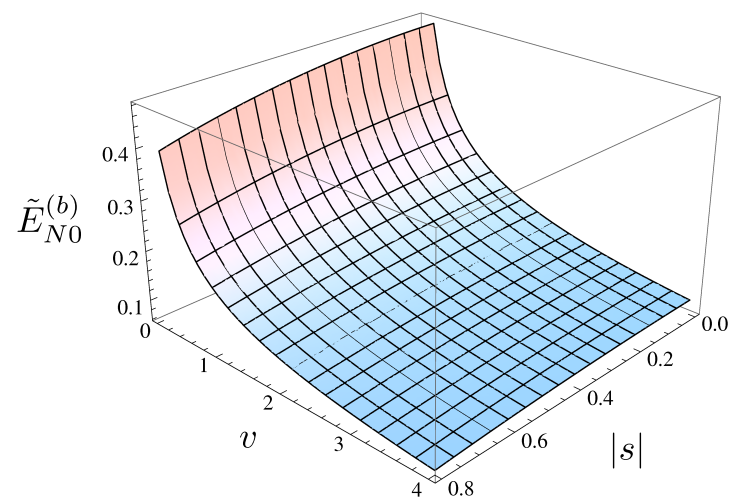

Figure 3: The dimensionless binding energy $\tilde{E}_{N 0}^{(b)}=E_{N 0}^{(b)} / \pi p d^{-1}$ calculated from (52), (27), (7) with $q=0.20$ for the ground state $(n=0)$ plotted as a function of the effective index of the corresponding subband $v=|N-\tilde{\sigma}|$ and the relative impurity position $s=\frac{2 x_{0}}{d}$ in the GNR of width $d$.

the armchair GNRs were studied in frame of the tight-binding model 35] and density functional theory [36], while Han et.al. [34] investigated experimentally the influence of the localized states in GNRs on the electron transport. The relation $E^{(b)} \sim d^{-1}$ including oscillations [35, 36] have been found to occur. The differences between the impurity states considered here and the exciton and localized states prevent us from a detailed quantitative comparison.

The Coulomb pattern of the energy levels (26) enables to introduce the effective Rydberg constant $R y_{N}$, the Bohr radius $a_{0 N}$ and the mass $M_{N}$ for the impurity electron in GNR

$$
R y_{N}=\frac{q^{2}|N-\tilde{\sigma}| \pi p}{2 d}, a_{0 N}=\frac{d}{\pi|N-\tilde{\sigma}| q}, M_{N}=\frac{|N-\tilde{\sigma}| \pi \hbar^{2}}{p d} .
$$

which additionally illustrate the physical reason of the bonding of the impurity electron, namely the quasi-1D geometry of the GNR. Note that the bound states arise at any finite width $d<\infty$. This result is qualitatively analogous to the effect of anti-diamagnetism caused by the influence of the magnetic field on the weakly bound atomic state. Demkov and Drukarev [19] considered the 3D potential well of small radius and depth to provide the capturing of the electron. It was shown that the arbitrarily weak magnetic field $B$ in- 
duces the bound electron state with the binding energy $E^{(b)} \sim a_{B}^{-2}\left(a_{B}=\right.$ $(\hbar / e B)^{1 / 2}$ is the magnetic length). The common reason for this is that the finite width $d<\infty$ and magnetic length $a_{B}<\infty$ transform the graphene monolayer and atomic structure, respectively, into the quasi-1D systems, which are more favorable to generate bound states. The dependencies $E^{(b)} \sim a_{B}^{-2}$ and $E^{(b)} \sim d^{-1}$ correspond to the different dispersion laws namely $E^{(b)} \sim p^{2}$ and $E^{(b)} \sim p\left(p \simeq \hbar / r\right.$ is the momentum) for the atomic $\left(r \simeq a_{B}\right)$ and $\operatorname{GNR}(r \simeq d)$ electron, respectively.

The dependence of the binding energy $E_{N n}^{(b)}$ (52) on the displacement of the impurity centre $x_{0}$ from the mid-point of the ribbon $x=0$ is contained in the corrections $\delta_{N n}\left(x_{0}\right)$ namely in the term $\ln D d^{-1}$ in eqs. (27) and (28), while $E_{N n}^{(b)}$ as a function of the effective number of the subband $|N-\tilde{\sigma}|$ is given by the sub-band threshold $\varepsilon_{N}$ (7) mainly and the term $\ln |N-\tilde{\sigma}|$ in the correction $\delta_{N n}$. The dimensionless binding energy $E_{N 0}^{(b)} / \pi p d^{-1}$ as a function of the effective quantum number $|N-\tilde{\sigma}|$ and relative displacement $x_{0} /(d / 2)$ for the ground $n=0$ state is depicted in Fig. 3. Clearly, the higher the subband i.e. the greater the value $|N-\tilde{\sigma}|$ is the less the binding energy $E_{N 0}^{(b)}$. Also the binding energy decreases when the impurity shifts from the mid-point of the ribbon towards the boundaries. The latter conclusion coincides with those obtained for the quantum well in Refs. [37, 38, 39, 40, 41.

The inter-band coupling shifts the strictly discrete excited Rydberg series $E_{N n}(N \neq 0)$ (26) calculated in single-subband approximation by an amount $W_{N n}$ (43) $N=1$, and (51) $N=-1$ and transforms them to the quasi-discrete levels of width $\Gamma_{N n}$ (42) $N=1$, and (50) $N=-1$. Note that the conclusions made on the base of the first and second excited subbands can be qualitatively extended to others. Since the resonant shifts $W_{N n} \sim q^{2}$ first are much less than the resonant widths $\Gamma_{N n} \sim q$ at $q \ll 1\left(W_{N n} \ll \Gamma_{N n}\right)$ and second the resonant shifts do not change the discrete character of the energy spectrum (26) we focus on the widths $\Gamma_{N n}$. It is clear from eqs. (42), and (50) that the widths $\Gamma_{N n} \sim \varepsilon_{N} \sim d^{-1}$ increase with decreasing the ribbons width $d$. Note that this dependence is opposite to that in a semiconductor narrow quantum well: the narrower the well is the less are the resonant widths [42, 38, 27. The reason for this is that in the quantum well the resonant width $\Gamma_{N n} \sim E_{N n}^{(b)}\left(E_{N n}^{(b)} / \Delta \varepsilon_{N}\right)^{2}$ where the impurity Rydberg constant $R y \simeq E_{N n}^{(b)}$ and the binding energy $E_{N n}^{(b)}$ do not depend on the well width $d$, while the inte-rband energy distance $\Delta \varepsilon_{N} \sim d^{-2}$ increases and consequently the resonant width decreases with the narrowing of the quantum well. For the ribbon $E_{N n}^{(b)} \sim \varepsilon_{N} \sim \Delta \varepsilon_{N} \sim d^{-1}$ (52) and the intersubband coupling do not depend on the ribbon width $d$ and $\Gamma_{N n} \sim E_{N n}^{(b)} \sim$ $\varepsilon_{N} \sim d^{-1}$.

The dependence of the resonant widths $\Gamma_{1 n}\left(x_{0}\right)$ (42), calculated in the double-subband approximation, on the position of the impurity centre $x_{0}$ is described by the coupling parameter $\gamma_{01}$ (37) and the corrections $\delta_{1 n}$ (27) and (28). The dependencies of the relative resonant widths $\Gamma_{1 n} / \varepsilon_{1}$ (42), on the dimensionless shift $s=2 x_{0} / d$ for the ground $n=0$ state are presented in Fig. 4 , 


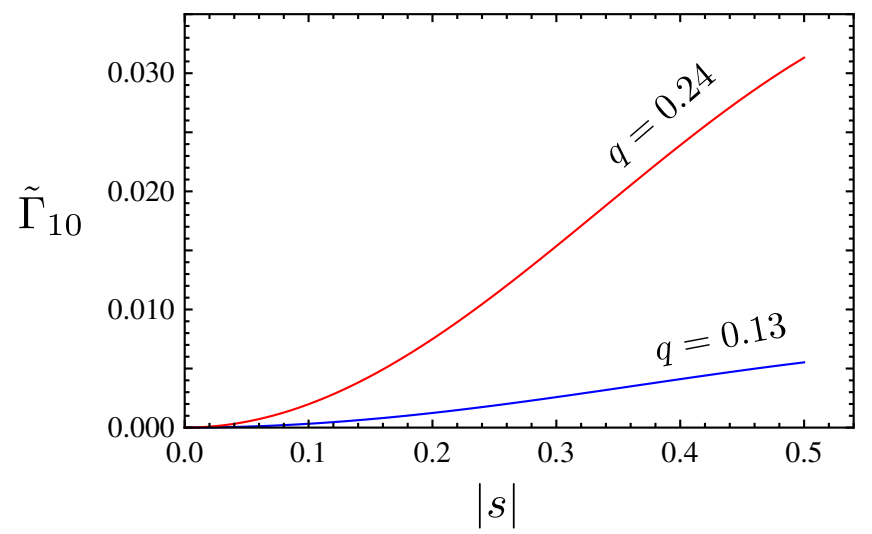

Figure 4: The resonant width $\Gamma_{10}$ (42) of the ground impurity state $(n=0)$ relatively to the corresponding threshold $\varepsilon_{1}$ (7) $\left(\tilde{\Gamma}_{10}=\Gamma_{10} / \varepsilon_{1}\right)$ versus the relative impurity position $s=\frac{2 x_{0}}{d}$ in the GNR of width $d$ providing the parameter $\tilde{\sigma}=0.3$. The parameter $q$ is taken to be $q=0.13 ; 0.24$.

in which the limitation on the parameter $s$ are caused by the condition imposed on $z_{0}$ placed below eq. (26). For the impurity positioned at the mid-point of the ribbon $x_{0}=0$ the resonant width and shift both vanish $\left(\Gamma_{1 n}(0)=W_{1 n}(0)=0\right)$ because of the even $x$-parity of the Coulomb potential $V(\vec{\rho})$ (2) in eq. (11) and opposite parities of the neighboring $N=0,1$ transverse $x$-states to give $V_{01}=\gamma_{0,1}=0$. Both in the quantum well and in the ribbon the shift of the impurities from their mid-points eliminates the even $x$-parity of the potential $V(\vec{\rho})(2)$ in eq. (11), that leads to the coupling between the $N=0$ and $N=1$ subbands. If the impurity displaces from the mid-point towards the boundaries $\left|x_{0}\right|=d / 2$ the resonant widths $\Gamma_{1 n}$ (42) monotonically increases. This correlates completely with the analogous dependence found for the impurity states in the semiconductor quantum well [42, 38, 27]. For small shifts $\alpha_{0} \ll 1$ in eq. (37) we obtain for the parameter $\gamma_{0,1}$ in eqs. (42) $\gamma_{0,1}=\left(\frac{2}{\pi}\right) \operatorname{Si}\left(\frac{\pi}{2}\right) \alpha_{0} \ll 1$ while for the impurity positioned close to the ribbon edge $x_{0} \simeq \frac{d}{2}$ we obtain $\gamma_{0,1}=\frac{1}{\pi} \operatorname{Si}(\pi)$ with $\operatorname{Si}\left(\frac{\pi}{2}\right)=1.37, \operatorname{Si}(\pi)=1.85$. Note that the zeroth width of the first excited $(N=1) n$ - series in case of the symmetrical $x_{0}=0$ impurity position is a consequence of the double-subband approximation. In the multisubband approximation the levels of the above mentioned series would acquire finite widths.

The dependence of the resonant widths (50) of the impurity states corresponding to the second excited subband $N=-1$ on the position of the impurity centre is completely different from that related to the first excited subband $N=1$. Equation (50) shows that contributions to the resonant widths $\Gamma_{-1 n}$ are caused by the coupling with the subbands $N=0\left(\sim \gamma_{0,-1}^{2}\right)$ (37) and $N=1\left(\sim \gamma_{1,-1}^{2}\right)$ (45). Note that the estimated contribution to 


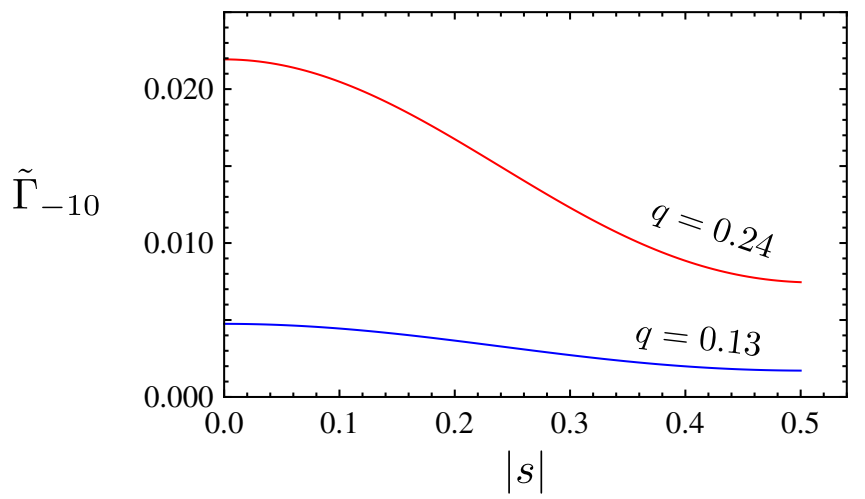

Figure 5: The resonant width $\Gamma_{-10}(150)$ of the ground impurity state $(n=0)$ relatively to the corresponding threshold $\varepsilon_{-1}$ (7) $\left(\tilde{\Gamma}_{-10}=\Gamma_{-10} / \varepsilon_{-1}\right)$ versus the relative impurity position $\frac{2 x_{0}}{d}$ in the GNR of width $d$ using the parameter value $\tilde{\sigma}=0.3$. The parameter $q$ is taken to be $q=0.13 ; 0.24$.

the resonant width $\Gamma_{-1 n}$ caused by the neglected coupling between the $N=$ 0 and $N=1$ subbands is of the order of $q \gamma_{01}^{2} \ll 1$. In the vicinity of the mid-point $\left(\left|x_{0}\right| \ll d / 2, \alpha_{0} \ll 1\right)$ the subband $N=1$ contributes mostly $\left(\gamma_{0,-1} \simeq 0, \gamma_{1,-1} \simeq-\frac{1}{\pi} \operatorname{Si}(\pi)\right)$, while for $\left|x_{0}\right| \simeq d / 2, \alpha_{0} \simeq \pi / 2$ both subbands contribute $\gamma_{0,-1} \simeq \frac{1}{\pi} S i(\pi), \gamma_{1,-1} \simeq \frac{1}{2 \pi} \operatorname{Si}(2 \pi)$. The position of the impurity $\bar{x}_{0}=\frac{d}{\pi} \bar{\alpha}_{0}$, at which the effects of the subbands $N=0$ and $N=1$ on the resonant width $\Gamma_{-1 n}$ are in balance is determined by the root $\bar{\alpha}_{0}$ of the equation

$$
\gamma_{1,-1}^{2} B_{1-1}=\gamma_{0,-1}^{2} B_{0-1} .
$$

to give the result $\bar{\alpha}_{0}=0.58, \bar{x}_{0}=0.37 \frac{d}{2}$. The coupling between the subbands $N=-1$ and $N=1$ provides the nonzero widths $\Gamma_{-1 n}$ and shifts $W_{-1 n}$ for any positions $x_{0}$ of the impurity. The width $\Gamma_{-10}$ as a function of the impurity shift $x_{0}$ is given in Fig. 5 demonstrating the monotonic drop within the same regions as those corresponding to Fig.4.

As mentioned above the presented method is valid under the conditions $q \ll 1$ for the excited impurity states $n=1,2, \ldots$ and $z_{0}(q) \ll 1$ (27) for the ground state $n=0$. Under these conditions the radius of the impurity state considerably exceeds the width of the GNR so that the ribbon is narrow compared to the impurity size. It follows from eq. (25) that a "big logarithm" can only hardly achieved [11, 12, 13, 14] i.e. the logarithmic approximation $|\ln q| \gg 1$ ensures the real smallness of $z_{0}$. However the previous calculations related to the ground state of the quasi-1D diamagnetic exciton 43 and present estimates show that a reasonably small parameter $q$ leads to values $z_{0}<1$, which provide a quite accurate and adequate description of the ground impurity state in GNR.

Taking into account possible experiments we estimate the expected electron 


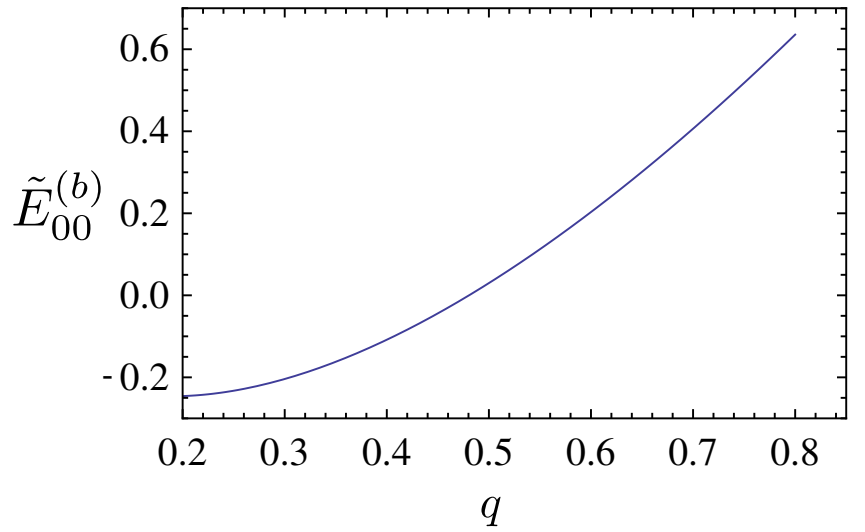

Figure 6: The dimensionless binding energy $\tilde{E}_{00}^{(b)}=E_{00}^{(b)} / \varepsilon_{0}$ of the quasi-classical ground state $(N=n=0)$ found from (55) for $E_{00}$ and from (7) with $\tilde{\sigma}=0.3$ for $\varepsilon_{0}$ versus the parameter $q$.

binding energy for the impurity centre placed at the middle point of the GNR of width $1 \mathrm{~nm}$ on the sapphire substrate as $E_{00}^{(b)} \simeq 160 \mathrm{meV}$ and on the $\mathrm{HfO}_{2}$ substrate as $E_{00}^{(b)} \simeq 68 \mathrm{meV}$. This is less than the data attributed to the $\mathrm{SiO}_{2}$ substrate $(\epsilon=3.9)$ because of the relatively small screening of the impurity potential. Also an estimate of the lifetimes $\tau_{N n}=\hbar / \Gamma_{N n}$ yields for the impurity positioned at the mid-point of the GNR $\tau_{-10} \simeq 0.21 \mathrm{ps}$ and $0.049 \mathrm{ps}$ for the $\mathrm{HfO}_{2}$ and sapphire substrate, respectively. For the $\mathrm{SiO}_{2}$ substrate the screening of the impurity attraction is less, the lifetime is reduced and therefore less favourable for a corresponding experimental observation. A shift of the impurity centre $\left|x_{0}\right| \simeq 0.4 d / 2$ generates lifetimes $\tau_{10}$ of the same order as $\tau_{-10}$ at $x_{0}=$ 0 . The electrons captured onto such short-lived trap states will most likely contribute to the dc transport. However, the high-frequency response of such electrons may reveal the signatures of localization.

Clearly, the above considered quasi-Rydberg series (26) do not cover the total set of discrete states. The oscillations of the wave functions (16) and (22) caused by the logarithmic term are an indicator of additional energy levels positioned below the series (26). Since the possible strong shift of these levels away from the threshold $\varepsilon_{N}$ is against the spirit of the employed adiabatic perturbation theory $(q \ll 1)$ describing the shallow energy levels we are limited to qualitative estimates based on the quasi-classical relativistic approach [9] and [4].

In an effort to elucidate the origin of this additional series let us consider the so called "logarithmic" energy levels governed by the logarithmic potential (14) taken for $d_{1}=d_{2}=d\left(x_{0}=0\right)$. These levels can be calculated from the Bohr-Sommerfeld quantization rule

$$
\int_{0}^{y_{0}} \mathcal{P}(y) d y=\pi \hbar\left(n+\frac{1}{2}\right) ; n=0,1,2, \ldots
$$


where $\mathcal{P}(y)$ and $y_{0} \ll d$ are the classical relativistic momentum and turning point, respectively, with

$$
\begin{aligned}
& \mathcal{P}^{2}(y)=\frac{1}{v_{F}^{2}}\left[\left(E-2 \frac{\beta}{d} \ln \frac{y}{d}\right)^{2}-\varepsilon_{N}^{2}\right] ; \\
& \mathcal{P}\left(y_{0}\right)=0 .
\end{aligned}
$$

Equation (53) admits an exact solution which provides for the energies

$$
E_{N n}=\frac{2 p}{d} q\left[\ln \frac{\left(n+\frac{1}{2}\right)}{|N-\tilde{\sigma}|}-\ln K_{1}\left(s_{N}\right)\right], s_{N}=\frac{\varepsilon_{N} d}{2 p q}=\frac{|N-\tilde{\sigma}| \pi}{2 q}
$$

where $K_{1}(s)$ is the modified Bessel function [30.

The binding energy $E_{N n}^{(b)}=\varepsilon_{N}-E_{N n}$ with $E_{N n}$ calculated from (55) reads $E_{N n}^{(b)} \sim q \ln q$ both for $q \ll 1\left(s_{N} \gg 1\right)$ and for $q \gg 1\left(s_{N} \ll 1,|\ln q| \gg 1\right)$. It follows that the weakness of the logarithmic singularity and smallness of the strength of the impurity potential $(q \ll 1)$ seems not to provide the bonding of the quasi-classical relativistic electron $\left(E_{N n}^{(b)}<0\right)$, while a sufficiently strong attraction $(q \leq 1)$ could produce a localized impurity state $\left(E_{N n}^{(b)}>0\right)$. The dependence of the binding energy of the quasi-classical ground state $(N=n=0)$ found from (55) on the parameter $q$ is depicted in Fig.6. The ground "logarithmic" level arises at the critical value $q_{0} \simeq 0.48$ and shifts towards lower energies to provide for the binding energy $0.1<E_{00}^{(b)} / \varepsilon_{0}<0.5$ for $0.54<q<0.74$. The above can be considered as no more than only a qualitative evidence of existence of such additional states in GNR that have transformed from the collapsed states in the graphene monolayer governed by the $2 \mathrm{D}$ impurity potential $\sim-r^{-1}[9]$. Though the "logarithmic" and quasi-Rydberg levels in principle correspond to the same region of the parameter $q<1$ any numerical comparison between the results for the quasi-Rydberg series based on the Dirac equation and those for the "logarithmic" levels derived from the quasi-classical method applied moreover to the ground state seems to be incorrect. The total set of the impurity states in GNR requires a further study of the equations (10) with the potential (11), having the logarithmic singularity in the vicinity of the impurity centre.

\section{Conclusion}

We have developed an analytical adiabatic approach to the problem of bound and meta-stable (Fano resonances) quasi-Coulomb impurity states in a narrow gaped armchair graphene nanoribbon (GNR). The width of the GNR is taken to be much less than the radius of the impurity. This adiabatic criterion implies a variable width of the GNR and simultaneously the smallness of the Coulomb interaction relative to the size-quantized energy induced by the GNR. 
The energy spectrum of the impurity electron is a sequence of the series of the quasi-Rydberg discrete and resonant states adjacent to the ground and excited size-quantized subbands, respectively. The binding energies and the resonant widths and shifts attributed to the inter-subband coupling are calculated in an explicit form in the single- and multi-subband approximation, respectively. The binding energies and the resonant widths both increase with decreasing the GNR width. As the impurity centre displaces from the mid-point of the GNR the binding energies decrease, while the resonant widths of the quasi-Rydberg series associated with the first/second excited sub-bands increase/decrease, respectively. Our analytical results are in complete agreement with those found by other theoretical approaches and in particular numerical studies. Estimates of the expected values show that the bound and meta-stable impurity states in GNR can be observed experimentally.

\section{Acknowledgments}

The authors are grateful to C. Morfonios for technical assistance. Financial support by the Deutsche Forschungsgemeinschaft is gratefully acknowledge.

\section{References}

[1] A. H. Castro Neto, F. Guinea, N. M. R. Peres, K. S. Novoselov, and A. K. Geim, Rev. Mod. Phys. 81109 (2009)

[2] O. Roslyak, G. Gumbs, and D. Huang. Phil. Trans. R. Soc. A 368, 5431 (2010)

[3] K. Namura and A. H. MacDonald, Phys. Rev. Lett. 98, 076602 (2007)

[4] N. M. R. Peres, F. Guinea and A. H. Castro Neto, Phys. Rev. B 73, 125411 (2006)

[5] R. B. Biswas, S. Sachdev, and D. T. Son, Phys. Rev. B 76, 205122046803 (2007)

[6] D. S. Novikov, Phys. Rev. B 76, 245435 (2007)

[7] V. M. Pereira, J. Nilsson, and A. H. Castro Neto, Phys. Rev. Lett. 99, 166802 (2007)

[8] A. V. Shytov, M. I. Katsnelson, and L. S. Levitov, Phys. Rev. Lett. 99, 236801 (2007)

[9] A. V. Shytov, M. I. Katsnelson, and L. S. Levitov, Phys. Rev. Lett. 99, $246802(2007)$

[10] L. D. Landau, and E. M. Lifshitz, Quantum Mechanics: Non-Relativistic Theory (Pergamon, London) 1981 
[11] A. M. Perelomov and V. S. Popov, Theor.Math.Phys. 4, 664 (1970)

[12] V. S. Popov, Phys.Atom.Nucl. Phys. 12, 429 (1970)

[13] V. S. Popov, JETP 60, 1228 (1971)

[14] Ya. B. Zeldovich and V. S. Popov, Sov.Phys.Usp.14, 673 (1972)

[15] K. S. Gupta, S Sen, Phys. Rev. B 78, 205429 (2008)

[16] K. S. Gupta, S Sen, Mod. Phys. Lett. A 24, 99 (2009)

[17] P. Harrison, Quantum Wells, Wires and Dots (Wiley New York, 2000)

[18] B. S. Monozon, P Schmelcher, Phys. Rev. B 79, 165314 (2009)

[19] Yu. N. Demkov, G. P. Drukarev, Sov. Phys. JETP-USSR 22, 182 (1966)

[20] L. V. Keldysh, JETP Lett. 29, 658 (1978)

[21] M. I. Katsnelson, K. S. Novoselov, and A. K. Geim,Nature Phys. 2, 620 (2006)

[22] M. I. Katsnelson, and K. S. Novoselov, Solid State Commun.143, 3 (2007)

[23] L. Brey and H. A. Fertig, Phys. Rev. B 73, 235411 (2006)

[24] U. Fano, Phys. Rev. 124, 1866 (1961)

[25] E. H. Hwang and S.Das Sarma, Phys. Rev. B 75, 205418 (2007)

[26] H. Hasegawa and R. E. Howard, J. Phys. Chem. Solids 21, 173 (1961)

[27] B. S. Monozon and P. Schmelcher, Phys. Rev. B 71, 085302 (2005)

[28] B. S. Monozon and P. Schmelcher, Phys. Rev. B 75, 245207 (2007)

[29] V. B. Berestetskii, E. M. Lifshitz, L. P. Pitaevskii, Quantum Electrodynamics, Butterworth-Heinemann, Oxford, Second Edition, (1982)

[30] Handbook of Mathematical Functions, edited by M. Abramowitz and I. A. Stegun (Dover, New York, 1972)

[31] Higher Transcendental Functions v.1, edited by H. Bateman and A. Erdelyi (Mc Graw-Hill Book Company, Inc., New York, Toronto, London 1953)

[32] R. G. NewtonScattering Theory of Waves and Particles (Springer, New York, 1982)

[33] J. Robertson, Eur. Phys. J. Appl. Phys.28, 265 (2004)

[34] M. Y. Han, J. C. Brant, and P. Kim, Phys. Rev. Lett. 104, 056801 (2010)

[35] Y. L. Jia, X. Geng, H. Sun, and Y. Luo, Eur. Phys. J. B 83, 451 (2011) 
[36] X. Zhu and H. Su, J. Phys. Chem. A 115, 11998 (2011)

[37] G. Bastard, Phys. Rev. B 24, 4714 (1981)

[38] A. Blom, M. A. Odnobludov, I. N. Yassievich, and K. A. Chao, Phys. Rev. B 68, 165338 (2003)

[39] C. Mailhiot, Y. -C. Chang, and T. C. McGill, Phys. Rev. B 26, 4449 (1982)

[40] K. Tanaka, M. Nagaoka, and T. Yamabe, Phys. Rev. B 28, 7068 (1983)

[41] R. L. Greene and K. K. Bajaj, Phys. Rev. B 31, 913 (1985)

[42] S. T. Yen, Phys. Rev. B 66, 075340 (2002)

[43] A. G. Zhilich and B. K. Kyuner, Sov. Phys. Semicond. 15, 1108 (1981)

[44] V. M. Pereira, V. N. Kotov, and A. H. Castro Neto, Phys. Rev. B 78, $085101(2008)$ 